\title{
Thermal Change of Depolarization Current in Polymer Electrets
}

\author{
Toshiaki TAKAMATSU and Eiichi FuKADA \\ The Institute of Physical and Chemical Research, Yamato, Saitama, Japan.
}

(Received September 10, 1969)

\begin{abstract}
Films of polyethylene, polytetrafluoroethylene and poly (vinylidene fluoride) were polarized to form electrets under a high static field at a high temperature. Electret films were cooled to near liquid nitrogen temperature and the depolarization current was measured as temperature increased. Several peaks of depolarization current were observed, which indicated the temperatures at which the change of thermal molecular motion or the phase transition in polymer structure took place. A peak was also observed near the temperature at which the electret was formed. The results show that the measurement of depolarization current in electret polymers could become a new method for thermal analysis of polymer properties.
\end{abstract}

KEY WORDS Electret / Depolarization Current / Polyethylene / Polytetrafluoroethylene / Poly (Vinylidene Fluoride) / Thermal Analysis / Molecular Motion /

Films of many kinds of polymers can be formed into electrets by applying a strong d.c. electric field at a high temperature and subsequently cooling to room temperature under an applied electric field. ${ }^{1,2}$ Accumulation of surface and volume charges as well as the orientation of dipoles in molecules bring about the formation of the electret state. If the electrodes on the surface of the film are connected to a sensitive electrometer, forming a short circuit between the two electrodes, a depolarization current is induced. The integrated amount of the depolarization current during the heating of the film gives the total electrical charge stored in the electret. ${ }^{3}$

In the present paper, many kinds of polymer films were transformed into electrets. The depolarization current of the electrets was measured in the course of increasing temperature from that of liquid nitrogen. Multiple peaks were then observed in the depolarization current $v s$. temperature curves, indicating a close connection between the temperature variation in the depolarization current and the thermal change in the polymer properties. The purpose of this paper is to describe quantitatively the experimental observations and to suggest some reasons for the existence of such multiple peaks in the depolarization current vs. temperature curve.

\section{EXPERIMENTAL METHOD}

Films of poly(vinylidene fluoride) with a thickness of $0.32 \mathrm{~mm}$, low density polyethylene (Alkathene 7F4), high density polyethylene (Sholex $6000-50)$ with a thickness of $0.1-0.3 \mathrm{~mm}$, and polytetrafluoroethylene (PTFE) with a thickness of $0.58 \mathrm{~mm}$ were used. Circular samples some $35 \mathrm{~mm}$ in diameter were cut out and areas about $25 \mathrm{~mm}$ across were painted centrally on both sides with silver paste, to serve as electrodes. A static high field between 0.5 and $2.5 \mathrm{kV}$ was applied between the two electrodes at different temperatures ranging from room temperature to near melting point. The films were then cooled down to about $-160^{\circ} \mathrm{C}$ with the strong electric field conditions sustained. Near the temperature of liquid nitrogen the films were short-circuited and the resultant depolarization current was detected by a Keithley 610B electrometer and recorded in an XY recorder. The thermoelectric voltage from the chromel-alumel thermocouple placed near the film was introduced to the $X$ axis of the recorder, while the depolarization current was led to the $Y$ axis. The temperature of the sample was gradually increased by the evaporation of the liquid nitrogen and the increase of the electric current in a heating coil. The rate of 


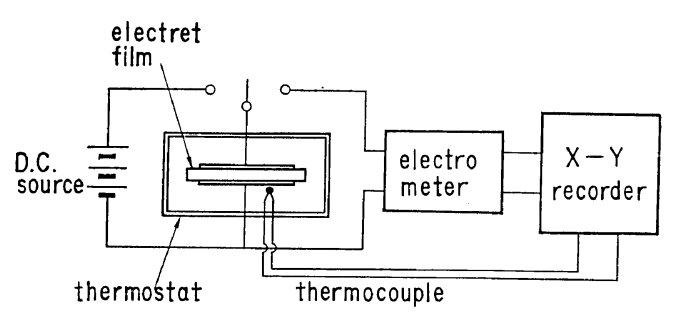

Figure 1. Schematic diagram of the apparatus for measuring the depolarization current of polymer electret films.

increase in temperature was approximately $1^{\circ} \mathrm{C} /$ $\min$.

\section{EXPERIMENTAL RESULTS}

Figure 2 illustrates the depolarization vs. temperature curves obtained for electrets of poly (vinylidene fluoride) $\left(\mathrm{PVF}_{2}\right)$ film. An electric voltage of $1.2 \mathrm{kV}$ was applied to the film at $72^{\circ} \mathrm{C}$ and $23^{\circ} \mathrm{C}$, the electric field being $3.9 \times 10^{4} \mathrm{~V} / \mathrm{cm}$. The polarizing temperature is denoted by $T_{p}$ in the graph. The sign of the depolarization current is the same as that of homocharge in the electret. This means that the positive charge flows back in the short circuit from the electrode of the sample which has been in contact with the positive side of the polarizing voltage. It can be clearly seen that the curves show a number of peaks at various temperatures. The glass transition temperature of $\mathrm{PVF}_{2}$ is about $-35^{\circ} \mathrm{C},{ }^{4}$ where the most distinct peak is observed. It is of interest to note that the peak at the highest temperature appears at about $89^{\circ} \mathrm{C}$ and $33^{\circ} \mathrm{C}$ when the polarizing temperature $T_{p}$ is $72^{\circ} \mathrm{C}$ and $23^{\circ} \mathrm{C}$, respectively. A large depolarization current is produced slightly above the temperature at which the electret has been formed.

Repeated experiments showed that the shape of the depolarization vs. temperature curve may change but the location of temperature for the peaks in the curves is quite reproducible. Figure 3 illustrates the peak temperature plotted against the polarizing temperature. The temperatures for five peaks are independent of the polarizing temperature. The highest peak temperature is linearly related to the polarizing temperature as described above.

Figure 4 shows the temperature dependence of

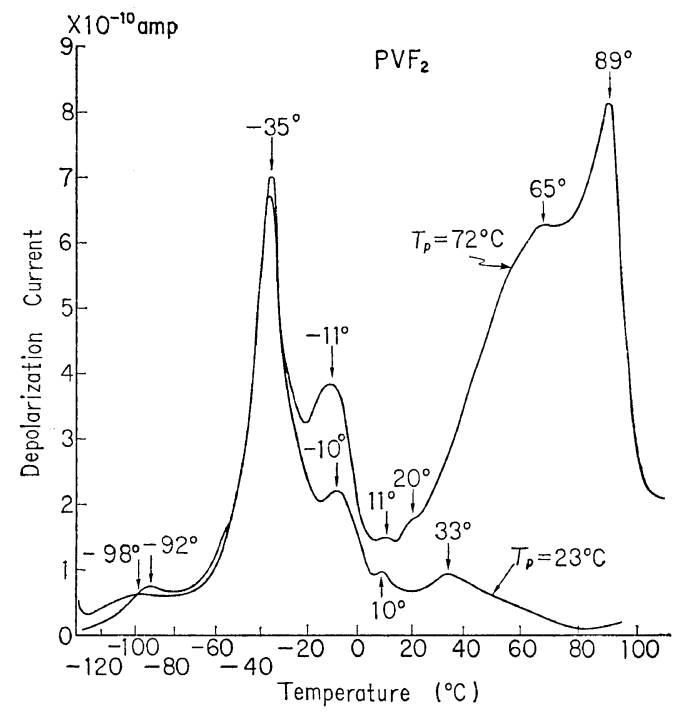

Figure 2. Examples of the depolarization current $v s$. temperature curves observed for polyvinylidene fluoride electret films. Polarizing field $E_{p}$ is $3.9 \times$ $10^{4} \mathrm{~V} / \mathrm{cm} . \quad T_{p}$ is the polarizing temperature.

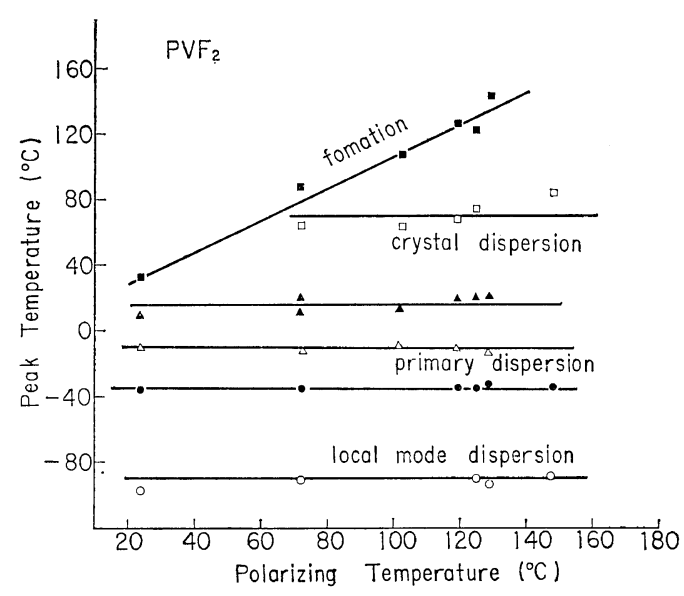

Figure 3. The peak temperature in depolarization current $v s$. polarizing temperature for poly (vinylidene fluoride) film.

the dynamic elastic modulus $E^{\prime}$ and loss modulus $E^{\prime \prime}$ for the $\mathrm{PVF}_{2}$ film, measured at a frequency of $30 \mathrm{~Hz}$ by a viscoelastorecorder developed in our laboratory. ${ }^{5}$ Peaks in the $E^{\prime \prime}$ curve are observed at about $-100^{\circ} \mathrm{C},-35^{\circ} \mathrm{C}$, and $60^{\circ} \mathrm{C}$. These absorptions are correlated to local relaxation and primary dispersion in the amorphous regions 


\section{Depolarization of Polymer Electrets}

and to crystalline dispersion. ${ }^{6,7}$ Corresponding peaks in the depolarization current are also observed in Figures 2 and 3. The peak temperatures at about $-10^{\circ} \mathrm{C}$ and $20^{\circ} \mathrm{C}$ are not assigned to any mechanism as yet. It is most interesting to note that the depolarization current may show peaks at the temperatures where the viscoelastic

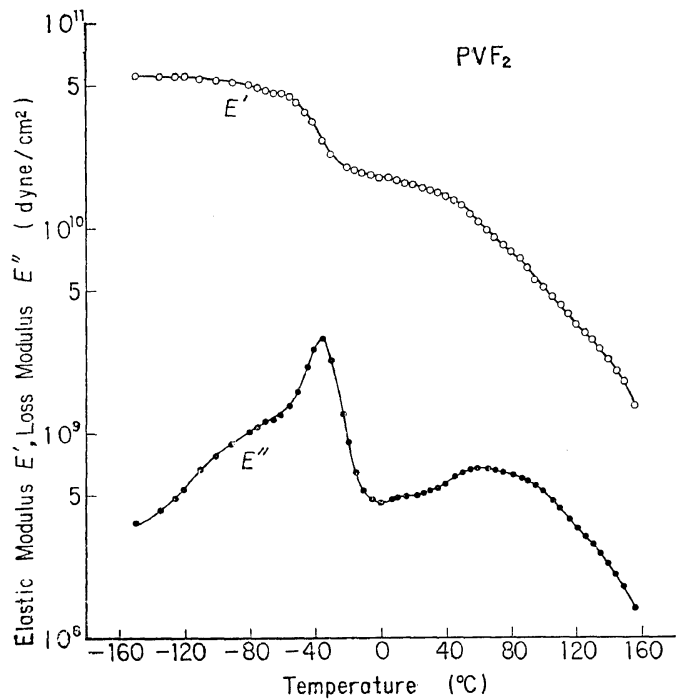

Figure 4. The dynamic elastic modulus and loss modulus $v s$. temperature for poly (vinylidene fluoride) film.

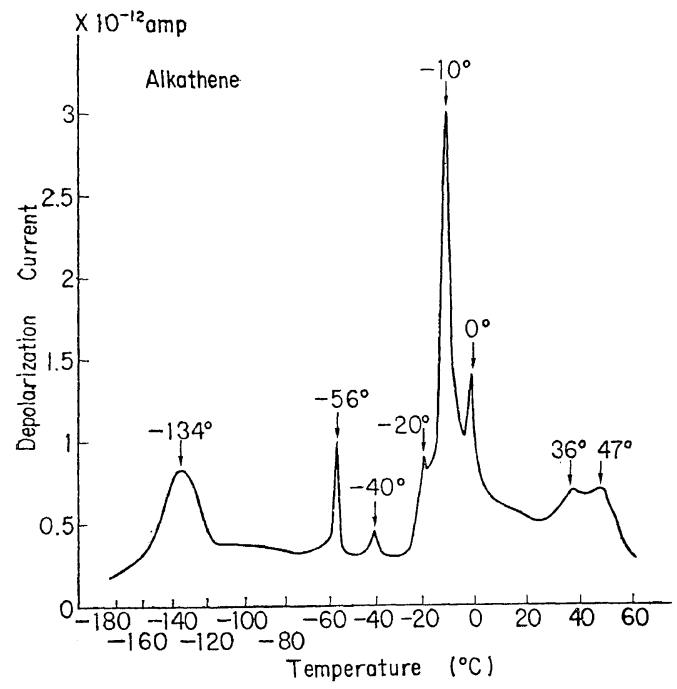

Figure 5. An example of depolarization current for low density polyethylene (Alkathene). $E_{p}=5$ $\times 10^{4} \mathrm{~V} / \mathrm{cm}, T_{p}=42^{\circ} \mathrm{C}$. measurement is not sensitive enough to give any indication.

Figures 5 through 7 illustrate examples of depolarization current $v s$. temperature curves in

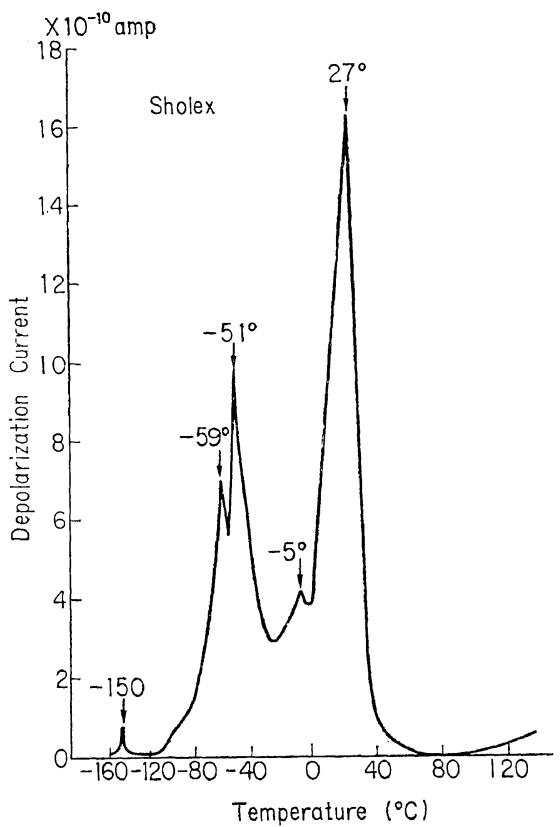

Figure 6. An example of depolarization current for high density polyethylene (Sholex). $\quad E_{p}=1.1$ $\times 10^{5} \mathrm{~V} / \mathrm{cm}, T_{p}=105^{\circ} \mathrm{C}$.

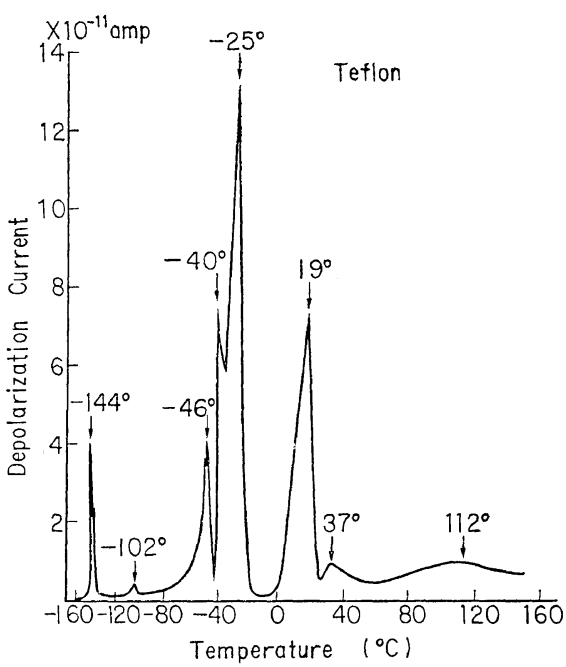

Figure 7. An example of depolarization current for polytetrafluoroethylene (Teflon). $E_{p}=2 \times 10^{4}$ $\mathrm{V} / \mathrm{cm}, T_{p}=109^{\circ} \mathrm{C}$. 


\section{T. TAKamatsu and E. Fukada}

an electret film of Alkathene (low density polyethylene), Sholex (high density polyethylene), and PTFE. Figures 8 through 10 show the peak temperatures plotted against the polarizing temperature for each polymer.

Figure 11 shows the temperature dispersion of

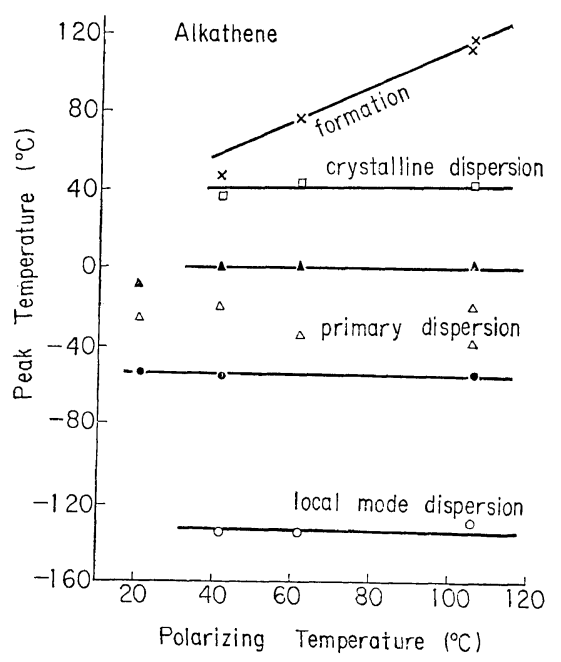

Figure 8. The peak temperature in depolarization current $v s$. polarizing temperature for low density polyethylene (Alkathene). $E_{p}=5 \times 10^{4} \mathrm{~V} / \mathrm{cm}$.

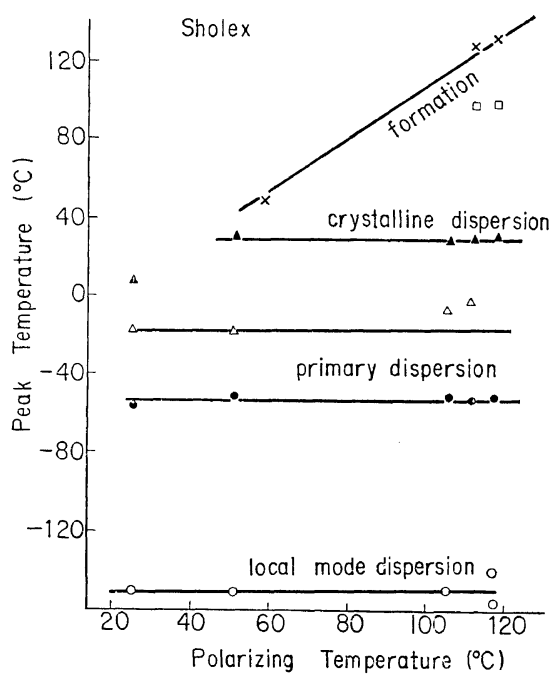

Figure 9. The peak temperature in depolarization current vs. polarizing temperature for high density polyethylene (Sholex). $E_{p}=1.1 \times 10^{5} \mathrm{~V} / \mathrm{cm}$. dynamic elastic modulus and loss modulus for Alkathene and Sholex. In Alkathene, the maxima of $E^{\prime \prime}$ are observed at about $-130^{\circ} \mathrm{C},-20^{\circ} \mathrm{C}$ and $50^{\circ} \mathrm{C}$, which are assigned to local mode relaxation, primary dispersion, and to crystalline

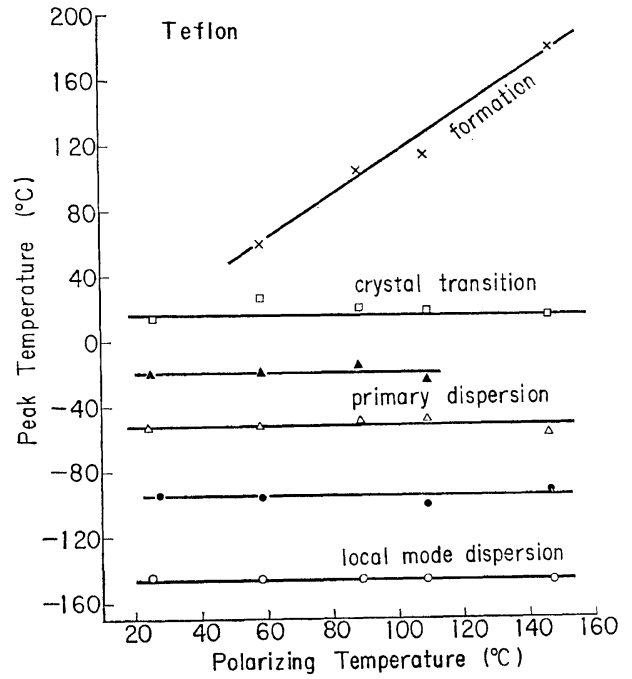

Figure 10. The peak temperature in depolarization current vs. polarizing temperature for polytetrafluoroethylene (Teflon). $E_{p}=2 \times 10^{4} \mathrm{~V} / \mathrm{cm}$.

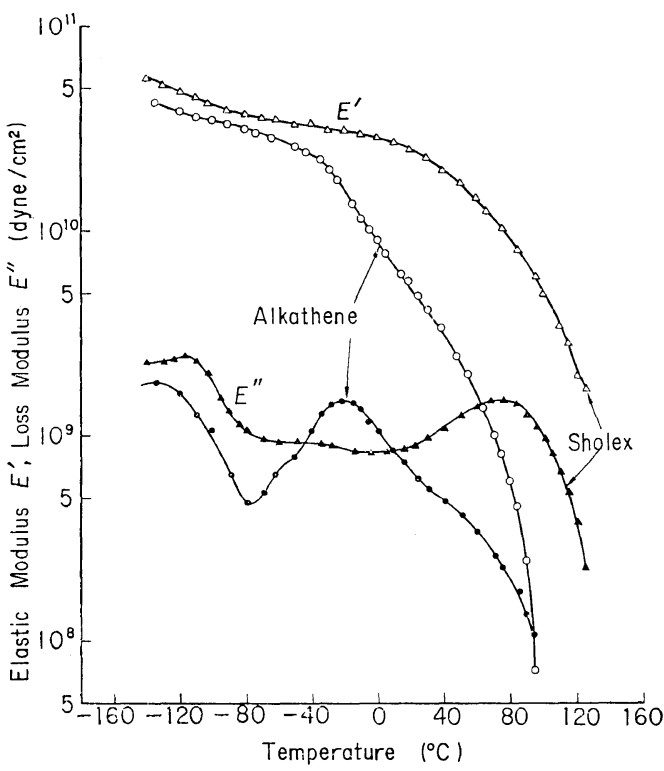

Figure 11. The dynamic elastic modulus and loss modulus at $30 \mathrm{~Hz} v s$. temperature for Alkathene and Sholex. 


\section{Depolarization of Polymer Electrets}

dispersion, respectively. ${ }^{8,9}$ The peaks at these temperatures are shown in the depolarization $v s$. temperatur ecurve in Figure 8. The maxima of $E^{\prime \prime}$ for Sholex are observed at $-120^{\circ} \mathrm{C},-40^{\circ} \mathrm{C}$, and $70^{\circ} \mathrm{C}$, which indicate local mode dispersion, primary dispersion and crystalline dispersion, respectively. ${ }^{8,9}$ The peaks at these temperatures may be seen from the depolarization vs. temperature curve in Figure 9.

\section{DISCUSSION}

Depolarization current exhibits many peaks with the rise in temperature. The reproducibility of the location of the peak temperature is fairly good as illustrated in Figures 3,8,9, and 10. Some scattered results were obtained in the temperature region above the glass transition temperature in polyethylenes. In all cases, the peak of the depolarization current was observed at a temperature slightly above that at which the electret was formed. During the formation of electrets under a strong electric field and at a high temperature $T_{p}$, a flow of ions occurs in the polymer film. Since the temperature $T_{p}$ is below melting point, the film keeps its solid shape. However a partial melting of crystallites will already have taken place. Crystallites of large size do not melt, but those of small size do. When the temperature of the film is lowered, a recrystallization of the small crystallites occurs. In the process ions become locally trapped and frozen in the crystalline regions. When the film is heated again to a temperature slightly in excess of $T_{p}$, the crystallites melt and the ions trapped inside such crystallites are released to flow freely. We may conclude therefore that the peak in the depolarization current at a temperature slightly above $T_{p}$ is due to ions trapped in the crystallites, the melting point of which lies approximately at $T_{p}$.

If the polymer film is heated to a high temperature approaching melting point and subjected to a strong static field, the electrons and ions should merge into the surface of the film from the external electrode. The transferred charge will then diffuse into the interior of the film and be trapped in various locations. The nature of the traps is not clear. They might be the holes or irregularities in amorphous regions, be defects or dislocations in crystalline regions, or be transient spots between crystalline and amorphous regions. The depth of the energy in the traps may be different depending on their nature. If the external electrodes are connected to the shortcircuit, the electric image force works on the trapped charges to draw them back out of the film. ${ }^{101}$ When the traps are located in the interior of crystallites, the melting of crystallites or the transition in crystalline phase will produce a large variation in the depth of energy of the trap. Therefore the charges held in such traps are released and contribute to the depolarization current. In the traps located in the amorphous regions, the energy depth of traps will be distributed over a certain range, but will be larger than the thermal energy of the trapped ions. If the temperature rises enough to give sufficient thermal energy to these ions to overcome the energy barrier in the traps, the diffusion of ions is enhanced under the electric field due to image force. The occurrence of thermal molecular motion will decrease the energy depth of traps and thus produce an increase in depolarization current. The fact that the sign of the depolarization current is the same as that of the homocharge of the electret can also be explained by these mechanisms, assuming that the charges are trapped predominantly near the surface.

In poly(vinylidene fluoride) and polyethylenes, the viscoelastic absorption measurements clearly indicate the three distinct dispersions; the local mode, primary, and crystalline dispersions. In the depolarization curve, peaks were observed at temperatures corresponding to the temperatures of viscoelastic absorption. However, in a certain range above the glass transition temperature, two or three peaks are usually observed in the depolarization current and an assignment of the origin for each peak temperature is not possible at the moment. Probably the strained or slightly oriented parts in amorphous regions would have a different activation energy for the diffusion of ions.

Many peaks in the depolarization current were also observed in PTFE as shown in Figure 10. From the transition map for PTFE, ${ }^{11}$ The temperature for local relaxation and primary dispersion at a very low frequency are estimated at approximately $-120^{\circ} \mathrm{C}$ and $-50^{\circ} \mathrm{C}$. The 
peak temperatures in the depolarization current in Figure 10 at $-140^{\circ} \mathrm{C}$ and $-50^{\circ} \mathrm{C}$ will correspond to these dispersions. A transition in crystalline phase takes place in PTFE at about $30^{\circ} \mathrm{C}$, which is also shown in the peak temperature in Figure 10. The mechanism for the peak temperatures at about $-20^{\circ} \mathrm{C}$ and $-90^{\circ} \mathrm{C}$ is not yet understood.

The peak heights in the depolarization current do not always remain the same if the measurements are repeated. The location of peak temperature is, however, reproducible irrespective of how the sample is treated. A close relationship between the peak temperatures for depolarization measurements and viscoelastic studies has been confirmed as demonstrated above. The sign of the depolarization current indicated that it was caused by the homocharge of the electret. Since the homocharge is distributed inside the sample, the release of the homocharge should be influenced by the thermal change of bulk properties of the polymer. We therefore suggest that the measurement of depolarization current in polarized polymer films can be used as a method for determining the temperatures where the transition of crystalline phase or the initiation of molecular motion in either crystalline or amorphous regions take place. This new method can detect with sensitivity the temperatures at which certain changes in polymer properties occur, even at temperatures where ordinary viscoelastic measurements are insensitive and ineffective. Differential thermal analysis
(DTA) is also frequently used for such a purpose. However, the thermal change of DTA curves obtained for the present samples was quite indistinct compared with that of the depolarization curves. According to our method the respective temperatures are shown as sharp peaks in the curve and are very easily detected. We propose that this new technique be called Electret Thermal Analysis.

\section{REFERENCES}

1. H. H. Wieder and S. Kaufman, J. Appl. Phys., 24, 156 (1953).

2. B. Gross, Charge Storage in Solid Dielectrics, Elsevier publishing Co., Amsterdam (1963).

3. B. Gross, J. Chem. Phys., 17, 866 (1949).

4. L. Mandelkern, G. M. Martin, and F. A. Quinn. Jr, J. Res. Nat. Bur. Stand., 58, 137 (1957).

5. M. Date and E. Fukada, Rep. Prog. Polym. Phys. Japan., 7, 183 (1964).

6. H. Sasabe and S. Saito, Rep. Prog. Polym. Phys. Japan., 11, 379 (1968).

7. M. Asahina, H. Kakutani, and K. Wada, Reprint of 16th Meeting for Discussion of Polymer Science (1967) p. 336.

8. Y. Wada, J. Phys. Soc. Japan., 16, 1226 (1960).

9. S. G. Turley and H. Keskkula, J. Polym. Sci., Part C. 14, 69 (1966).

10. T. Furukawa, Y. Uematsu, K. Asakawa, and Y. Wada, J. Appl. Polym. Sci., 12, 2675 (1968).

11. Y. Ohzawa and Y. Wada, J. Appl. Phys. Japan., 3, 436 (1964). 